\title{
Effect of Thermal Radiation on Unsteady Gravity Flow of a Power-Law Fluid with Viscous Dissipation through a Porous Medium
}

\author{
A.W. Ogunsola, B.A. Peter \\ Dept. Of Pure And Applied Mathematics,LadokeAkintola University Of Technology, Ogbomoso,Nigeria
}

\begin{abstract}
$\overline{\text { Abstract:In this work, we studied the effect of thermal radiation on unsteady gravity flow of a power-law fluid }}$ with viscous dissipation through a porous medium. The modified Darcy's law is considered together with the equation of energy transfer in such media. It is assumed that the viscosity is temperature-dependent. We transformed the governing partial differential equations into ordinary differential equations in terms of a suitable similarity variable. We employed Galerkin weighted residual method to solve the resulting non-linear equations. The results show the effects of thermal radiation parameter and other physical parameters involve in the system of flow.
\end{abstract}

Keywords: Unsteady gravity flow, weighted residual method, Power-law fluid and thermal radiation.

\section{Introduction}

The unsteady and steady flow of Newtonian and non-Newtonian fluids in porous media in which the main driving force is gravity has attracted the attention of many scientists in the recent times. Due to the increase in the production of heavy crude oils, and elsewhere where materials whose flow behaviour due to shear stress and shear rate cannot be characterized by Newtonian relationships; it has become necessary to have an adequate understanding of the rheological effects of non-Newtonian fluid flows and, as a result, a new stage in the evolution of fluid dynamic theory is in progress.

Salem[1] examined the effects of variable viscosity, viscous dissipation and chemical reaction on heat and mass transfer flow of MHD micropolar fluid along a permeable stretching sheet in a non-Darcian porous medium. Abdel[2] studied the effects of variable viscosity and thermal conductivity on unsteady MHD flow of non-Newtonian fluid over a stretching porous sheet. Braginsky et al [3] examined temperature dependent variable thermal conductivity of porous structures. Cortell [4] investigated on unsteady gravity flows of a power-law fluid through a porous medium. He analyze the flow in two direction, one side both thinning and thickening of the fluids and on the other hand, two different types of solutions, for the case of a gravity flow generated by the injection of a power-law fluid at the well into an empty reservoir of an infinite extent. The effect of variable viscosity and thermal conductivity of micro polar fluid in a porous channel in the presence of magnetic field was considered by Gitima [5]. Hayat et al [6] analyzed stretching flow of casson fluid with variable thermal conductivity. Unsteady temperature field of a power-law fluid with variable thermal conductivity was investigated by Olajuwon [7].

Similarity solutions to some gravity flows of non-Newtonian fluids through a porous medium were studied by Pascal and Pascal [8]. Singh[9] considered the effects of viscous dissipation and variable viscosity effects on MHD boundary layer flow in porous medium past a moving vertical plate with suction. Ogunsola and Ayeni [10] examined the temperature distribution of an Arrheniusly reacting unsteady flow through a porous medium with variable permeability. Szeri and Rajagopal [11] examined the flow of a Non-Newtonian fluid between heated parallel plates. Howarth [12] numerically considered various aspect of the Blasius flat-plate flow problem. Sparrow and Cess [13] studied the effect of magnetic field on free convection heat transfer on isothermal vertical plate. Krishnendu et al [14] considered similarity solution of mixed convective boundary layer slip flow over a vertical plate. Hayat et al [15] they considered the effect of joule heating and thermal radiation in flow of third grade fluid over a radiative surface. Tomer et al [16] examined the effect of variable viscosity on convective heat transfer along an inclined plate embedded in porous medium with an inclined magnetic field.In this work, we considered the effect of thermal radiation parameter on the flow model.

\section{Governing Equations}

In this work, unsteady gravity flow with viscous dissipation of a power-law fluid through a porous medium with thermal radiation is considered. The governing equations are continuity, momentum and energy equations. The governing equations are analyzed using a similarity transformation in terms of a similarity 
variable. The model parameters which arise from the flow system are viscous dissipation parameter $p_{0}$, thermal conductivity parameter $k_{0}, \mathrm{Re}$, Reynolds number, $\mathrm{Pr}$, Prandtl number, and $\mathrm{Br}$, Brinkman number.

Considering a two dimensional flow in the $x-z$ plane where the free surface is a streamline at a point on the surface, we expressed the flow by a modified Darcy's law.There are three basic differential equations of fluid motion:

$\frac{\partial(h u)}{\partial r}=-\Phi \frac{\partial h}{\partial t}$

The local continuity condition (Huppert 1982a; Huppert \& Woods 1995) is

$\frac{1}{r} \frac{\partial(r h u)}{\partial r}=-\Phi \frac{\partial h}{\partial t}$

(Mass balance Equation)

where $\Phi=g / 2 m$, $\Phi$ being the porosity of the porous medium, which is assumed to be constant in both space and time.

$$
\begin{aligned}
& u=-\left(\frac{k \rho}{\mu_{e f}}\right)^{\frac{1}{n}} \frac{\partial h}{\partial s}\left|\frac{\partial h}{\partial s}\right|^{\frac{1-n}{n}} \quad \text { (Modified Darcy's law-Momentum Equation) } \\
& \rho c_{p} \frac{\partial T}{\partial t}=\frac{1}{r} \frac{\partial}{\partial r}\left(r k \frac{\partial T}{\partial r}\right)+\mu\left(\frac{\partial u}{\partial r}\right)^{2}-\left(\frac{\partial q_{r}}{\partial y}\right)
\end{aligned}
$$

The appropriate initial and boundary conditions for this work are

$$
h(0, t)=h_{1}, h(\infty, t)=h_{0}, T(r, 0)=T_{0}, T(0, t)=T_{1}, T(\infty, t)=T_{0} \quad t>0
$$

where $k$-Thermal conductivity, $\rho$-Density, $C_{p}$-Specific heat at constant pressure, where $\sigma^{*}$ is the StefanBoltzmann constant, $k_{1}$ is the mean absorption coefficient, $\mu$-Dynamic viscosity, $\mu\left(\frac{\partial h}{\partial r}\right)^{2}$ is the viscous heating effect, $V_{r}$-Component of velocity in the radial direction, $\mu_{e f}$-Effective viscosity, $n$ - Dimensionless Power-law index, $e^{T}$-Thermal expansion, $k_{0}$ - The thermal conductivity of the fluid, $\gamma$-thermal expansion exponent, $T_{0}$-Initial temperature and it is the reference temperature, $T$-Temperature within the boundary layer, $T_{1}, T_{2}, \ldots . . T_{\infty}$ - Temperature at the plate, $p=\rho g h, \eta$-Apparent viscosity ,i.e. Similarity variable parameter, $f$-is a dimensionless stream function, $m_{0}$ or $k_{1}$-Flow consistency index, $\theta$-Dimensionless temperature.

The first two terms on the right hand side of the equation represent the heat conduction and viscous dissipation term.

\section{Method Of Solution}

$$
\begin{aligned}
& \frac{1}{r} \frac{\partial(r h u)}{\partial r}=-\Phi \frac{\partial h}{\partial t} \\
& u=-\left(\frac{k \rho}{\mu_{e f}}\right)^{\frac{1}{n}} \frac{\partial h}{\partial r}\left|\frac{\partial h}{\partial r}\right|^{\frac{1-n}{n}}
\end{aligned}
$$

Taking the positive value of $\frac{\partial h}{\partial r}$ since the velocity which is expressed in terms of the fluid height in the porous medium is non-negative we have

$$
u=-\left(\frac{k \rho}{\mu_{e f}}\right)^{1 / n}\left(\frac{\partial h}{\partial r}\right)^{\frac{1}{n}}
$$


Substituting equation (8) into (6) we obtain

$$
\frac{1}{r} \frac{\partial}{\partial r}\left(\frac{\partial h}{\partial r}\left(\frac{\partial h}{\partial r}\right)^{\frac{1-n}{n}} h r\right)=\Phi\left(\frac{\mu_{e f}}{k \rho}\right)^{\frac{1}{n}} \frac{\partial h}{\partial t} \quad \text { (Cylindrical coordinate) }
$$

We introduce the following non-dimensional variables

$t^{\prime}=\frac{t}{t_{0}}, \theta(r, t)=\frac{T-T_{0}}{T_{1}-T_{0}}, r^{\prime}=\frac{r}{R}, h^{\prime}=\frac{h}{h_{0}}$

$t=t^{\prime} t_{0}, T=\theta(r, t)\left[\left(T_{1}-T_{0}\right)+T_{0}\right], r=r^{\prime} R$

We seek similarity solution of the form

$h_{1}=h_{2} t^{\alpha}, h(r, t)=h_{2} t^{\alpha} f(\eta) ; \eta=r t^{\beta}, \theta(r, t)=t^{\lambda} \phi(\eta)=t^{\lambda} g(\eta)$

where $\alpha, \beta \in \mathfrak{R}$ and $f$ is a function of $\eta$ only

Substituting equation (11) into (9) we obtain

$$
\begin{aligned}
& \frac{d}{d \eta}\left(\eta f f^{\prime}\left|f^{\prime}\right|^{\frac{1-n}{n}}\right)=a^{2} \eta\left(\alpha f-\frac{n+\alpha}{n+1} \eta \frac{d f}{d \eta}\right)(1-\theta \lambda) \\
& f(0)=1, f(\infty)=0, f^{\prime}(\infty)=1, \phi(0)=1, \phi(\infty)=0
\end{aligned}
$$

We now consider the energy equation through a porous medium with thermal radiation and density depending on temperature as follows:

$$
\begin{aligned}
& \rho c_{p} \frac{\partial T}{\partial t}=\frac{1}{r} \frac{\partial}{\partial r}\left(r k \frac{\partial T}{\partial r}\right)+\mu\left(\frac{\partial u}{\partial r}\right)^{2}-\left(\frac{\partial q_{r}}{\partial y}\right) \\
& q_{r}=-\frac{4 \sigma^{*}}{3 k_{1}} \frac{\partial T^{4}}{\partial y}, \rho(T)=\rho_{0}\left[1-\lambda_{0}\left(T-T_{0}\right)\right]
\end{aligned}
$$

substituting the dimensionless variable Equations(10)\& (14) into (15) and dropping the primes, we obtain

$$
\frac{\partial \theta}{\partial t}(1-\theta \lambda)=-\gamma k_{0} e^{-s \theta} \frac{B r}{\operatorname{Re} \operatorname{Pr}}\left(\frac{\partial \theta}{\partial r}\right)^{2}+\frac{1}{\operatorname{Pr}} e^{-s \theta} \frac{1}{r} \frac{\partial \theta}{\partial r}+\frac{1}{\operatorname{Pr}} k_{0} e^{-s \theta} \frac{\partial^{2} \theta}{\partial(r)^{2}}\left(1+\frac{4}{3} R_{d}\right)+p_{0}\left[\frac{\partial}{\partial r}\left(\frac{\partial h}{\partial r}\right)^{\frac{1}{n}}\right]^{2}
$$

where $\quad \gamma_{1}=\gamma\left(T_{1}-T_{0}\right), p_{0}=\frac{\mu h_{0}^{2 / n}}{\rho_{0} c_{p}\left(T_{1}-T_{0}\right)}\left(\frac{k \rho}{R \mu_{e f}}\right)^{2 / n}, R_{3}=\frac{t_{0}}{R^{2}} e^{\gamma_{1} T_{0}}, \lambda=\lambda_{0}\left(T_{1}-T_{0}\right), R_{d}=\frac{4 \sigma^{*} T_{\infty}^{3}}{k^{*} K}$,

$$
B r=\frac{u_{0}^{2} \mu R_{4}}{k_{0}\left(T_{1}-T_{0}\right)}, \operatorname{Pr}=\frac{\mu \rho_{0} c_{p}}{R_{3} k_{0}}, \operatorname{Re}=\frac{l_{0} u_{0} \rho_{0}}{\mu}, R_{4}=\frac{e^{-\gamma_{1} T_{0}} k_{0} t_{0} \rho_{0}\left(T_{1}-T_{0}\right)^{2}}{l_{0} R^{2} \mu u_{0}}, \text { Peclet }=\operatorname{Re}^{*} \operatorname{Pr}
$$

$\theta(r, 0)=0, \theta(0, t)=1, \theta(\infty, t)=0$

substituting equation (11) into (16) we obtain

$$
(1-\theta \lambda) \beta \eta \phi^{\prime} e^{\gamma_{1} \phi}=\left[-\frac{B r}{P e} \gamma\left(\phi^{\prime}\right)^{2}+\frac{1}{\operatorname{Pr}}\left(\eta^{-1} \phi^{\prime}+\phi^{\prime \prime}\left(1+\frac{4}{3} R_{d}\right)\right)\right]+p_{0} e^{\gamma_{1} \phi}\left[\frac{d}{d \eta}\left(f^{\prime}\right)^{\frac{1}{n}}\right]^{2}
$$

Since the initial temperature may not necessarily be zero we have

$$
\phi(0)=1, \phi(\infty)=0, \eta \in(0, \infty)
$$

We now proceed to solve Equations (12) and (19) subject to (13) numerically using Galerkin-weighted residual method as follows:

$$
\text { let } f=\sum_{i=0}^{2} A_{i} e^{y}, \phi=\sum_{i=0}^{2} B_{i} e^{(-1 / 4) y}
$$

The results are presented in Figures 1-6 


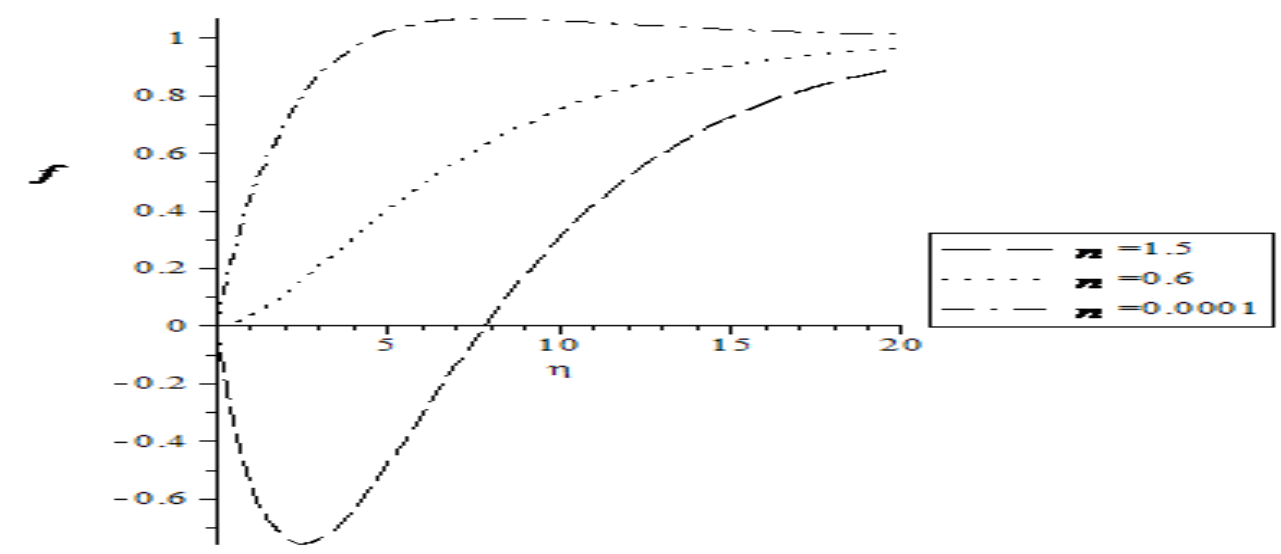

Figure 1: Graph of the velocity function $f$ for various value of porous radial flow with constant viscosity when $\alpha=0.5$.

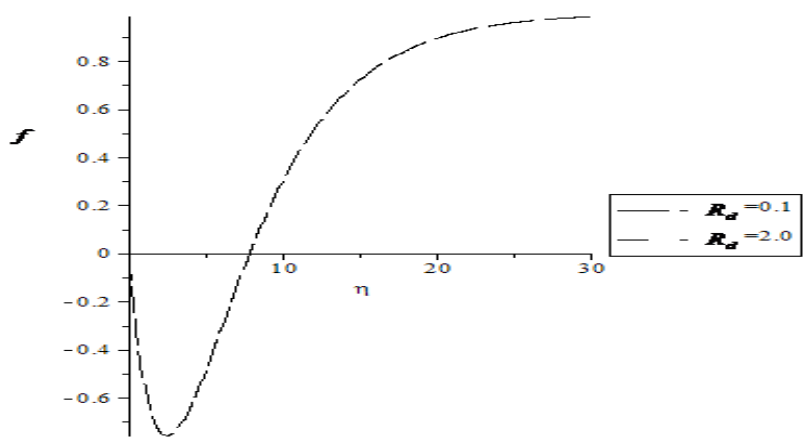

Figure 2: Graph of the velocity function $f$ for various value of porous radial flow with constant viscosity when $\alpha=n==0.5$.

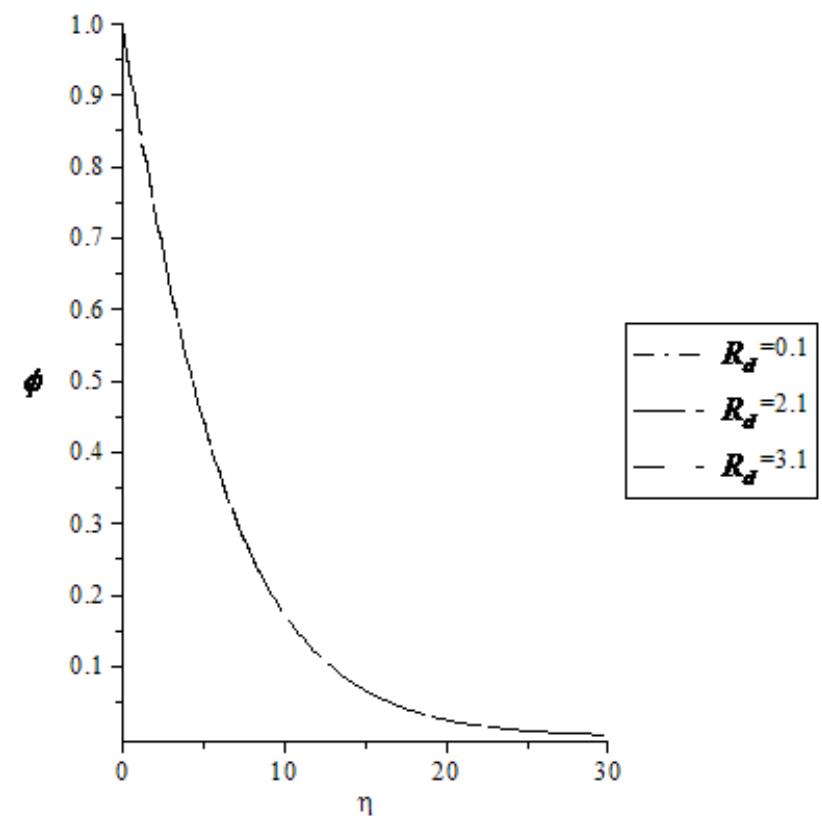

Figure3: Graph of the temperature function $\phi$ for various value of $g$ when $\beta=1.5, n>0, \operatorname{Pr}=0.25, \operatorname{Re}=1.0, B r=0.75$. 


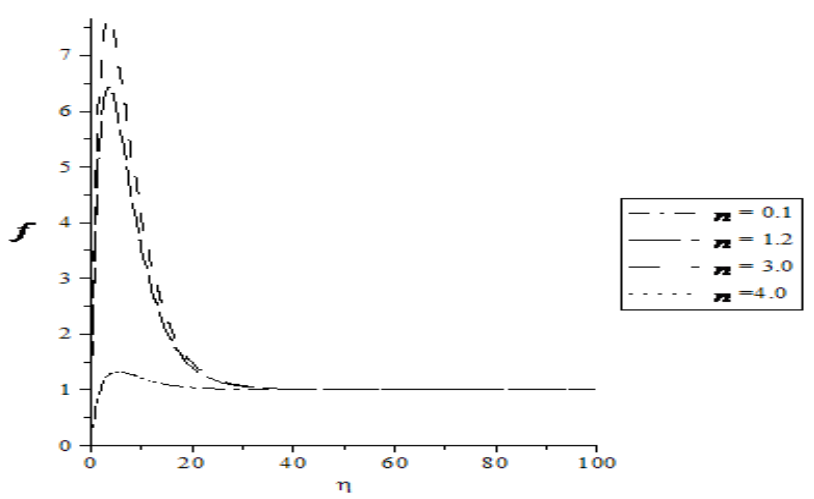

Figure 4: Graph of the velocity function $f$ for various value of porous radial flow with constant viscosity when

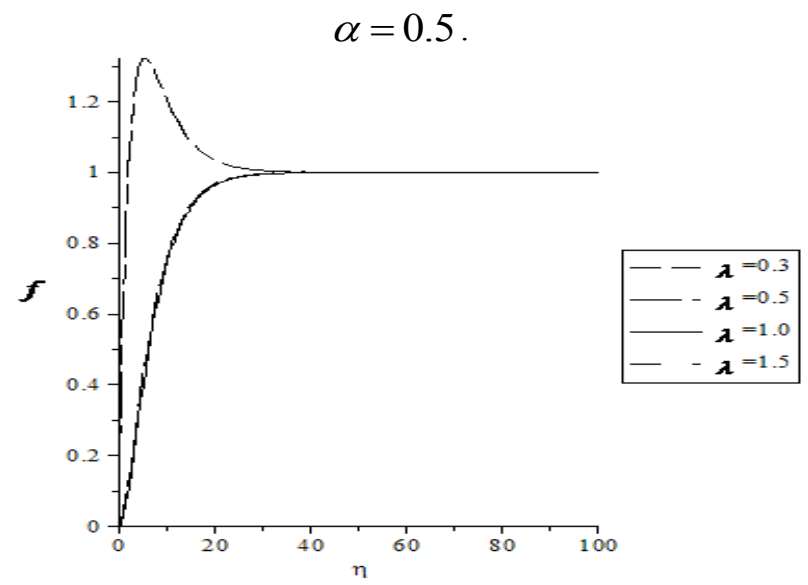

Figure 5: Graph of the velocity function $f$ for various value of porous radial flow with constant viscosity when

$$
\alpha=0.5 \text {. }
$$

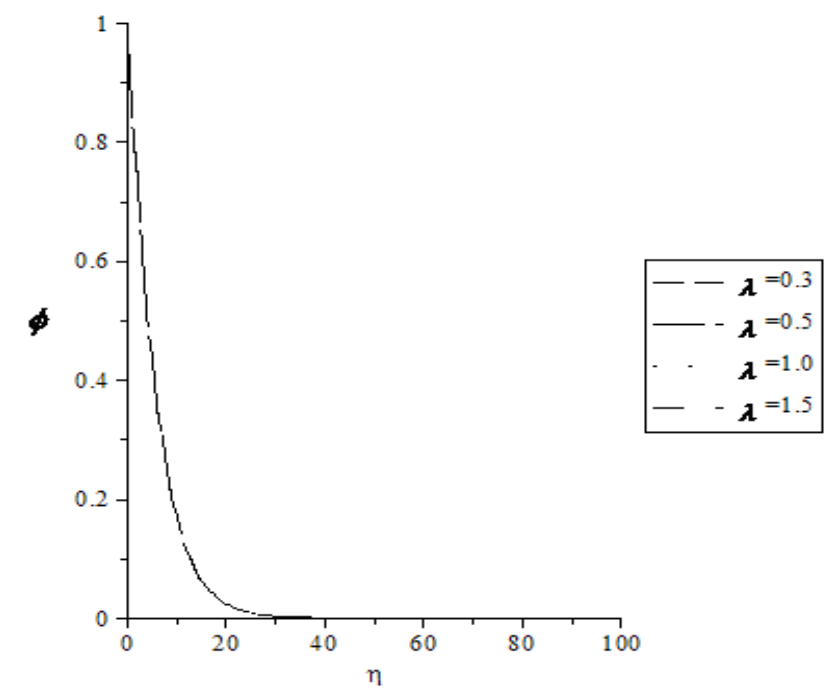

Figure6: Graph of the temperature function $\phi$ for various value of $g$ when

$$
\beta=1.5, n>0, \operatorname{Pr}=0.25, \operatorname{Re}=1.0, B r=0.75 .
$$

\section{Discussion of Results}

\section{Discussion of Results and Conclusion}

We have considered a suitable model of unsteady variable thermal conductivity gravity flow of a power-law fluid with thermal radiation parameter through a porous medium. The result from Figure 1shows that as power-law index decreases the velocity express in terms of stream function $f$ increases monotonically. The result from Figure 2 shows that as thermal radiation parameter increases the fluid velocity increases monotonically. From Figure 3 the results show that the temperature decreases monotonically with increase in 
each of Brinkman number, Prandtl number, Reynolds number, and thermal radiation parameter. From Figure 4 the result shows that the velocity profile increases monotonically as density parameter increases. From Figure 5 the result shows that the velocity profile decreases monotonically as power-law index decreases. The result from Figure 6 shows that the temperature profile decreases with increase in density parameter and other physical parameters. Physically, increase in the Prandtl number is due to an increase in the viscosity of the fluid. On the other hand, from analytical and numerical calculations we also see that the parameter $n, R_{d}, \operatorname{Pr}, \operatorname{Re}, p_{0}, \beta, \lambda$ and $B r$ affect the flow characteristics significantly.

\section{Conclusion}

A set of non-linear coupled differential equations governing the fluid mass and energy transfer is solved numerically for various parameters. It is noted that the influence of thermal conductivity parameter on the flow system is to increase the fluid temperature. It can be concluded that the increase in physical parameters i.e. Reynolds number, Prandtl number, Brinkman number and Peclet number; and thermal radiation parameter leads to a corresponding decrease in the viscosity of the fluid. This will be of great importance for the field engineers in various processes of oil recovery.

\section{References}

[1] Kumar, B. and Prasad (2014): Pulsatile flow through a porous medium. J. of Appl. Fluid Mech.,Vol.7,No.1,pp63-74,2014.

[2] Vajravelu, K, Prasad, K.V. Santh, S.R. and Umesh,V. (2014): Fluid flow and heat transfer over a permeable stretching cylinder. J. of Applied Fluid Mech.,Vol.7,No.1,pp 111-120,2014.

[3] Salem, A.M. (2013):The effects of variable viscosity, viscous dissipation and chemical reaction on heat and mass transfer flow of MHD micropolar fluid along a permeable stretching sheet in a non-Darcian porous medium. Journal of Mathematical Problems in Engineering,Vol.,2013.

[4] GitimaPatowanj (2012): Effect of variable viscosity and thermal conductivity of micro polar fluid in a porous channel in the presence of magnetic field. Int. J. of Basic Sciences and Social Sciences, Vol.3, pp.69-77.

[5] Rafael CortellBataller (2008): Unsteady gravity flows of a power- law fluid through a porous medium. Applied Maths.and Computation 196 pp356-362.

[6] Ogunsola, A.W. and Ayeni, R.O.(2007):Temperature distribution of an Arrheniusly reacting unsteady flow through a porous medium with variable permeability. Research Journal of Applied Sciences, Issue 2, Vol.4,pp 400-403.

[7] Pascal, J.P. and Pascal, H. (1993): Similarity solutions to some gravity flows of non-Newt. fluids through a porous medium. Int. J. Non-Linear Mech.38, 157.

[8] Hupper. H.E. \& Woods, A.W.(1995):Gravity-driven flows in porous layers. J. Fluid Mech., Vol.292,pp 55-69.

[9] Singh (2013): Viscous dissipation and variable viscosity effects on MHD boundary layer flow in porous medium past a vertical plate with suction. Int. Journal of Engineering Science and Technology. http:/www.waset.org/journals/ijens/v4-3-30. Accessesed 11th June,2013.

[10] Ogunsola, A.W. and Ayeni, R.O.(2007).Temperature distribution of an Arrheniusly reacting unsteady flow through a porous medium with variable permeability. Research Journal of Applied Sciences, Issue 2(4), 400-403.

[11] A.Z. Szeri and K.R. Rajagopal, Flow of a Non-Newtonian fluid between heated parallel plates. Int. J. Non-Linear Mechanics, Vol.20.No. 2. 91-101, 1985.

[12] Howart L, on the solution of the Laminar boundary layer equations. Proc Roy Soc London A 1938;164:547-557.

[13] Sparrow EM and Cess RD, The effects of magnetic field on free convection heat transfer. Int. J. Heat Mass Transfer,3(1961),264774.

[14] Krishnendu Bhattacharyya, Swati Mukhopadhyay and G.C.Layek, Similarity solution of mixed convective boundary layer slip flow over a vertical plate. Ain Shams Engineering Journal 4(2013),299-305.

[15] Hayat Tasawar, AnumShafig and Ahmed Alsaedi (2014) Effect of Joule heating and thermal radiation in flow of third grade fluid over radiative surface. Nonlinear Analysis and Applied Mathematics Research Group, Faculty of Science, Saudi Arabia.9(1), 1-12. http:/www.plosone.org. Accessesed March,2014.

[16] Tomer N.S., Phool Singh and Manoj Kumar (2010). The effect of variable viscosity on convective heat transfer along an inclined plate embedded in porous medium with an inclined magnetic field. Int. J. of Engineering and Natural Sciences. http:/www.waset.org/journals/ijens/v4-3-30. Accessesed 6th July,2013. 